\section{Cahiers de Narratologie}

Analyse et théorie narratives

10.2 | 2001

La voix narrative

\title{
Chronique de deux morts annoncées : «El Hijo » (Horacio Quiroga) et Plenilunio (Antonio Muñoz Molina)
}

\section{Christine Pérès}

\section{(2) OpenEdition \\ Journals}

Édition électronique

URL : http://journals.openedition.org/narratologie/10252

DOI : 10.4000/narratologie. 10252

ISSN : 1765-307X

Éditeur

LIRCES

Édition imprimée

Date de publication : 1 janvier 2001

Pagination : 351-360

ISBN : 2914561032

ISSN : 0993-8516

Référence électronique

Christine Pérès, «Chronique de deux morts annoncées : «El Hijo » (Horacio Quiroga) et Plenilunio (Antonio Muñoz Molina) », Cahiers de Narratologie [En ligne], 10.2 | 2001, mis en ligne le 01 janvier 2001, consulté le 11 juin 2020. URL : http://journals.openedition.org/narratologie/10252 ; DOI : https://doi.org/10.4000/narratologie.10252 


\title{
CHRONIQUE DE DEUX MORTS ANNONCÉES : « EL HIJO » (HORACIO QUIROGA) ET PLENILUNIO (ANTONIO MUÑOZ MOLINA)
}

\author{
Christine PÉRÈS \\ Université de Toulouse
}

Vouloir comparer deux œuvres aussi différentes que «El hijo » et Plenilunio - que tout sépare à première vue (genre, époque, continent) - peut sembler surprenant. L'une' est un conte publié en 1927 par l'écrivain uruguayen Horacio Quiroga. L'autre 2 , un roman écrit en 1997 par l'Espagnol Antonio Muñoz Molina. Toutefois le conte et le dernier chapitre du roman sont unis par un double dénouement et la thématique commune d'une mort annoncée (ou pressentie) et cependant génératrice de suspense et d'angoisse. Notre étude de la voix narrative se fondera sur la définition classique donnée à ce terme par Genette ${ }^{3}$. Après avoir présenté le double dénouement de ces textes, nous montrerons qu'il s'avère impossible d'y analyser la voix narrative sans impliquer le mode : en effet, la relation entre la voix et la perspective narrative est mise au service d'une stratégie conférant au lecteur un rôle différent, ce qui nous conduira à nous interroger sur les fondements idéologiques qui président à ce changement.

\section{I - Voix narrative et double dénouement}

Les deux textes sont pris en charge par un narrateur non personnel. Cependant, chez Muñoz Molina, il s'agit d'une narration rétrospective au passé, apparemment plus objective, tandis que chez Quiroga, l'emploi du présent est l'indice

\footnotetext{
${ }^{1}$ Horacio QUIROGA, Cuentos, Madrid, Cátedra, 1997.

2 Ántonio MUÑOZ MOLINA, Plenilunio, Madrid, Alfaguara, 1997.

${ }^{3}$ Gérard GENETTE, Figures III, Paris, Seuil, 1972.
} 
d'une narration simultanée. Dans les deux cas, le texte se fonde sur une relation duelle entre l'émetteur et le récepteur et joue constamment avec " un déficit programmé et consubstantiel d'information ${ }^{4}$. Mais chez Quiroga, le narrateur adopte l'allure d'un témoin et feint de partager le déficit d'information du lecteur tandis que chez Muñoz Molina il fait clairement état du surplus d'information qu'il détient et semble disposé à le partager avec le récepteur. Ces textes qui jouent avec la mort se caractérisent en outre par un double dénouement auquel le lecteur peut adhérer, explicite chez l'un, implicite chez l'autre.

Le conte «El hijo » est formé de deux parties séparées par une double ligne de points : elle sert à visualiser un double dénouement et à mettre en évidence une stratégie narrative de manipulation du lecteur soulignée par la disproportion typographique entre les deux mouvements du texte. La diégèse a pour cadre la forêt amazonienne de Misiones, située aux confins du Paraguay et de l'Uruguay. La première partie relate le départ pour la chasse du fils, sa disparition, la quête angoissée du père - victime d'hallucinations lui présentant son fils mort - et les heureuses retrouvailles finales. La seconde, très brève, inverse complètement ce dénouement heureux, car elle se clôt sur l'expression " muerto desde las diez de la mañana » et révèle que la rencontre finale n'était qu'une simple hallucination. L'essentiel du texte est constitué par la longue quête du père caractérisée par l'angoisse croissante de découvrir à chaque instant le cadavre de son fils. La pulsion essentielle qui l'anime est donc celle de savoir : elle a trait à une connaissance essentielle qui est celle de la vie ou de la mort, découverte mise en valeur par la dernière expression du conte. Mais, curieusement, bien que la mort du fils soit présentée jusqu'à la fin comme une tragédie inéluctable, elle surprend le lecteur. Le décalage final entre le destinataire - qui découvre la vérité - et le protagoniste inconscient de la réalité des faits - montre qu'il convient

4 Jean ALSINA et Michelle DÉBAX, «"Suspender”. Ensayo de descripción de un hacer informativo", in Alsina, Jean (éd.) Suspens/Suspense, Cric § Ophrys, 1993, p. 22. 
Chronique de deux morts annoncées :

« El hijo» (H. Quiroga) et Plenilunio (A. Muñoz Molina)

d'étudier les mécanismes de création de la tension en relation avec le maniement de la perspective narrative.

L'avant dernier chapitre de Plenilunio se clôt sur une scène présentée en focalisation interne: Susana Grey contemple depuis sa fenêtre son amant, l'inspecteur, qui s'éloigne dans la rue lorsque soudain un homme à l'attitude étrange s'approche de lui par derrière; elle pousse un cri tandis que retentit le premier coup de feu. Dès l'avant-dernier chapitre, le lecteur est averti que plusieurs coups vont être tirés. Le dernier chapitre se caractérise par une rupture de la continuité temporelle et un changement de perspective narrative : une analepse interne qui nous ramène une fraction de seconde avant le coup de feu est présentée selon la perspective de l'inspecteur. Alors que dès l'ouverture du chapitre le lecteur, conduit par la voix narrative à épouser le point de vue du protagoniste, fait l'expérience de la mort, la fin du texte laisse planer le doute : tombé sous les balles d'un membre de l'E.T.A., l'inspecteur perd connaissance dans les bras de sa maîtresse venue à son secours. Est-il mort ou simplement inconscient? Ce dénouement unique témoigne d'un maniement de l'art du suspense et d'une fin ouverte puisqu'à l'ignorance du protagoniste répond celle du lecteur. Beaucoup plus ambigu que le double dénouement du conte, il repose sur le maniement de la voix narrative, sur ses capacités de manipulation et s'ouvre plus encore sur le monde du lecteur, auquel il revient de prolonger le récit pour lui donner une des deux fins possibles postulées par le texte.

\section{II - Le rôle du lecteur}

Ces textes posent le problème du décodage du récit par le lecteur et nous conduisent à nous interroger sur la façon dont l'expression infléchit la saisie du contenu. Chez Quiroga, le destinataire est l'objet d'une manipulation rhétorique tandis que dans Plenilunio un rôle de coauteur lui est dévolu. 
Chez Quiroga, le premier dénouement représente - en apparence du moins - une restauration de la situation initiales, qu'une comparaison entre l'incipit et la première fin permet de mettre en évidence. D'où l'effet de surprise créé par le second dénouement.

L'incipit s'ouvre sur une vision du cadre spatiotemporel prise en charge par un narrateur à la troisième personne assumant un rôle de témoin. Tout célèbre I'harmonie régnant entre personnage et nature : par le biais de deux focalisations internes qui servent à personnifier le paysage et à évoquer l'état d'esprit du père, un parallélisme se construit entre le personnage et le cadre naturel, osmose que parachève l'emploi du verbe "abrir » pour les qualifier tous deux. Le bref dialogue entre le père et son fils met en évidence la docilité de l'enfant qui acquiesce face à la tendre autorité paternelle, docilité mise aussi en évidence par les verbes déclaratifs qui accompagnent le dialogue. Dans les deux premières répliques, la focalisation est différente selon le personnage : externe pour le fils, interne pour le père. L'emploi du déictique à valeur anaphorique («abreviando en esa frase ») évoque par sa valeur allusive un savoir que les deux personnages partagent ( $y$ que su hijo comprende »), de même que les lecteurs qui viennent de lire cette phrase, de sorte qu'au-delà de la complicité entre père et fils s'instaure aussi une complicité entre narrateur et narrataire. Dans les répliques suivantes, la focalisation est externe pour les deux personnages : à aucun moment, le fils n'est présenté en focalisation interne.

En raison de ses ressemblances avec l'incipit, le premier dénouement semble une restauration de la situation initiale, conférant au conte une circularité apparente : réunion du couple initial, bref dialogue au style direct, verbes déclaratifs suggérant un parallélisme entre le père et le fils, gestes de tendresse, répétition du verbe " comprender ». Le lexique du bonheur, présent dans l'incipit, réapparaît. Pour la première

5 Paul LARIVAILlE, "L'analyse (morpho)logique du récit ", Poétique, $\mathrm{n}^{\circ}$ 19, 1974. 
Chronique de deux morts annoncées :

« El hijo » (H. Quiroga) et Plenilunio (A. Muñoz Molina)

fois, le fils est présenté en focalisation interne. Comme le récit est pris en charge par un narrateur non personnel qui adopte la plupart du temps la perspective du père, associant ainsi le destinataire à la quête, l'utilisation de la focalisation interne pour le fils provoque l'adhésion du lecteur annulant la tension créée antérieurement. Le narrateur adopte aussi la perspective du père. Après la vision subjective des deux personnages, une phrase en focalisation externe présente le couple réuni (p. 355) : la neutralité du narrateur qui semble se cantonner dans un rôle de témoin, sert alors à tromper le lecteur et à lui dissimuler le caractère illusoire de cette félicité retrouvée.

Certains éléments suggèrent toutefois que la scène finale diffère de l'incipit. La phrase qui fait suite au dialogue, « Nimio detalle después de todo », caractérisée par son ambiguïté, peut être considérée dans un premier temps comme une pensée du père au style direct libre mais la présence de l'adjectif « nimio » dans plusieurs fragments antérieurs pris en charge par le narrateur (p. 353, 354) montre qu'il s'agit d'un commentaire de ce dernier sur la situation finale. D'autre part, l'adéquation entre personnage et nature a complètement disparu car le père épuisé est écrasé par un soleil de plomb : la nature - toujours ouverte - s'est changée de paradis en enfer. Enfin, un jeu s'instaure entre les articles et les possessifs. La valeur universelle de l'article indéfini « un $»^{6}$, suivi du possessif «el nuestro », témoigne d'un savoir partagé par le narrateur - devenu pour un temps personnel-et le narrataire :

«Las fuerzas que permiten entregar un pobre padre alucinado a la más atroz pesadilla tienen también un límite. Y el nuestro siente que las suyas se le escapan, cuando ve bruscamente desembocar de un pique lateral a $\underline{s u}$ hijo. A $\underline{u n}$ chico de trece años bastábale ver desde cincuenta metros la expresión de $\underline{s u}$ padre sin machete dentro del monte, para apresurar el paso con los ojos húmedos. » (p. 355)

6 Michel CAMPRUBI, Etudes fonctionnelles de grammaire espagnole, Toulouse, PUM, 1990, pp. 23-24. 
Mais l'utilisation des articles et des possessifs diffère selon les personnages : « un - el nuestro - su » v/s « un - su ». Dans le cas du père, on passe de l'universel au particulier par le biais du possessif « el nuestro » qui instaure une connivence entre narrateur et narrataire tandis que dans le cas du fils, on reste au stade de l'universel puisque le pronom possessif est absent. Ce n'est pas la vision interne du fils mais celle de n'importe quel fils devant l'inquiétude paternelle. Cette tromperie passe inaperçue lors de la première lecture car ce fils « universel » se voit attribuer la perspective du fils particulier du texte : la vision du père épuisé sortant de la forêt sans machete. $\mathrm{Ce}$ "un» universel figure dans des passages antérieurs présentés selon la perspective du père (p. 352). Le mot «hombre » présent dans l'étiquette du personnage souligne la rupture de la relation filiale. Il apparaît pour la première fois lorsque retentit le coup de feu annonciateur de la mort de l'enfant, événement présenté comme un fait négligeable («nimio ») au moyen d'une ellipse temporelle occultée par une pause descriptive célébrant l'harmonie entre le père et la nature. Le temps paraît s'effacer entre cet instant et midi, heure à laquelle, absorbé par son travail, il prend conscience que son fils n'est pas rentré.

La manipulation temporelle est encore plus évidente dans le dernier chapitre de Plenilunio, formé de trois paragraphes de plus en plus courts : le premier, long de trois pages, couvre seulement une fraction de seconde et s'achève lorsque retentit le premier coup de feu, le second - une page correspond aux coups de feu suivants, le troisième - six lignes - relate l'évanouissement de l'inspecteur. Les deux derniers couvrent une durée indéterminée, la première vraisemblablement de quelques minutes, une durée bien supérieure donc à celle de l'analepse, la seconde de quelques secondes sans doute. Le dernier chapitre se caractérise donc par une accélération du tempo narratif propice au suspense, mise en évidence par la présence de l'inverseur sémantique ${ }^{7}$ «pero » en tête du second paragraphe : le lecteur a l'impression que le temps qui s'était suspendu devant l'imminence de

${ }^{7}$ Ibid., p. 49. 
« El hijo » (H. Quiroga) et Plenilunio (A. Muñoz Molina)

la mort se remet en marche, avant de se suspendre définitivement. La dernière phrase se caractérise par une adéquation entre le temps de l'histoire et celui du récit.

L'analepse interne du premier paragraphe s'ouvre sur une série d'anachronies au second degré dont la fonction diffère. Une prolepse interne informe le lecteur sur les conséquences du premier coup de feu et semble traduire la vision ultime d'un mourant : depuis le sol, l'inspecteur aperçoit les chaussures de son agresseur éclaboussées de sang. Ensuite, quatre analepses internes de même portée, mises en évidence par l'anaphore du verbe "salió », créent un effet de dilatation temporelle : chacune d'elles présente l'inspecteur au moment où il sort de la maison de sa maîtresse. La quatrième nous ramène au point de départ du chapitre, une fraction de seconde avant que ne retentisse le coup de feu. La phrase qui clôt le premier paragraphe ne peut qu'attirer l'attention du lecteur car la tension y atteint son point extrême en présentant le personnage face à la mort :

«Pensó con estupor, con inesperada tranquilidad «así que ésta era la cara del que iba a matarme », y en el interior de ese segundo que no llegaba a terminar comprendió que la verdadera inminencia de la muerte sólo puede conocerla quien está a punto de morir, que ninguna otra sensación en la vida se le parece o la anuncia : la calma, el asombro, la silenciosa detención del tiempo. » (p. 483-484)

L'emploi de deux imparfaits dans la phrase au style direct est l'indice d'une manipulation rhétorique du narrateur: on attendrait deux présents ou un présent suivi d'un imparfait. Cet usage d'un double imparfait serait plus approprié dans un texte au style indirect libre. Cette rupture dans la logique des temps employés maintient l'ambiguîté sur l'issue de la scène évoquée dans cette phrase qui dit à la fois la vie et la mort. Le style direct suggère que le danger de mort appartient au passé (comme si le personnage se dédoublait et contemplait l'événement a posteriori) tandis que le présent de vérité générale introduit par le verbe "comprendió » annonce sa prise de conscience de l'imminence de l'issue fatale, comme un écho, une confirmation de celle du narrateur. Cette issue fatale ne semble faire aucun doute pour ce dernier qui, quelques lignes 
plus haut, décrit au moyen du présent de vérité universelle ce que l'on éprouve à l'instant ultime, mais cette mort présente toutes les caractéristiques de celle qui menace l'inspecteur : présence d'une lumière aveuglante, dilatation temporelle, balle tirée dans la nuque (« ... pero... dé en los ojos. », p. 483). Le suspense ne porte donc pas sur la mort du personnage mais sur sa possible prise de conscience de son imminence, comme en témoigne cette phrase où le narrateur semble soudain en proie au doute :

«... es probable que si no hubiera empezado a volverse y a intuir el peligro no habría llegado a saber lo que estaba a punto de ocurrirle, y tal vez habría muerto sin enterarse siquiera de que iba a morir... » (p. 482-483)

De même, lorsque le deuxième paragraphe nous ramène au moment annoncé par la prolepse interne du premier, le récit donne l'impression de revenir sans cesse sur ses traces et le lecteur en sait à présent apparemment autant que le narrateur, ce qui réactive le suspense. Le récit semble s'arrêter sans cesse aux portes de la mort, ce que va venir confirmer la fin du texte. La phrase contenant la citation au style direct écrite avec un double imparfait fait office de clé de lecture, elle dessine les contours de l'architecture générale du chapitre qui dit à la fois la vie et la mort, trait que l'on retrouve de manière récurrente dans bon nombre de phrases, aussi bien celle qui ouvre le chapitre que celle qui le clôt.

Le champ lexical de la mort s'y construit avec des termes relatifs au bruit et au silence, à la vue et à l'ombre, à la léthargie et à l'instinct, au calme, à l'étonnement, au sang, à l'arrêt du temps. L'absence d'inverseur sémantique entre le deuxième et le troisième paragraphe montre qu'on reste dans une logique de vie, mais le champ lexical de la mort est toujours présent. Cette ambiguïté persiste jusque dans la dernière expression "un sueño afiebrado de turbiones de sangre y sirenas de ambulancias », dans laquelle les flots de sang annoncent la perte de la vie et les sirènes d'ambulance l'arrivée des secours. Cette dialectique entre vie et mort apparaît dans l'attitude même du personnage : il dit la vie (cf. ses dernières paroles) mais la façon dont il étreint Susana dit à nouveau sa peur de la perdre (p. 482 // p. 485). Muñoz Molina laisse une porte de sortie à son personnage : suspens 
Chronique de deux morts annoncées :

«El hijo » (H. Quiroga) et Plenilunio (A. Muñoz Molina)

et suspense vont de pair. Comme les séries américaines " interactives » où la dernière image nous offre une image du héros inconscient frappé par balle (Dallas, Les héritiers) et où en fonction des souhaits exprimés par les sondages, on choisit ou non de le ressusciter lors de l'épisode suivant, le lecteur se voit attribuer un rôle actif. Il est curieux de constater que, alors que rien ne permet de trancher en faveur de la vie ou de la mort de l'inspecteur, le lecteur prête généralement une fin heureuse au roman. Peut-être tout simplement parce que ce texte refuse de dire la mort en la différant sans cesse. Cette incertitude est aussi l'indice d'un jeu intertextuel avec les structures du roman du XIX ${ }^{\mathrm{e}}$, ces "romans-tombeaux » où l'épisode final de la mort du héros était sans équivoque, fait que dénonce Muñoz Molina ${ }^{8}$.

La présence obsédante de la mort sous-tend ces textes qui la disent sans la décrire et qui se caractérisent par une inversion du schéma de communication posé au départ : chez Quiroga, le narrateur abandonne son rôle de simple témoin et affiche un savoir qu'il transmet au lecteur sur le mode de la révélation; chez Muñoz Molina, le narrateur qui semblait détenir un savoir choisit de se taire. Dans les deux cas, la mise en place du second dénouement est préparée tout au long du texte, mais ses finalités diffèrent. Dans le premier cas, il constitue le coup de théâtre final précédant la résolution de la tension, dans l'autre cette tension perdure. Chez Quiroga, cette mort qui surprend dans la fiction le lecteur et les personnages se veut le reflet de celle qui nous surprend dans la vie. La présence indéniable du cadavre - dernière image qu'emporte le lecteur - sert aussi à configurer une vision du monde dans laquelle le triomphe de la mort paraît sanctionner la défaite de l'Homme face à la Nature et l'échec d'un père, leitmotive obsédants chez cet écrivain. Chez Muñoz Molina au contraire, son absence tend à suggérer que la mort est l'Indicible, l'Immontrable. Dès que le policier s'évanouit, le texte fait silence. Et cette mort est pour le lecteur l'Impensable, l'Inacceptable. C'est là que réside la différence fonda-

8 Antonio MUÑOZ MOLINA, Las apariencias, Madrid, Alfaguara, 1995 , p. 269. 
mentale entre les deux textes. Celui de Muñoz Molina doit être resitué dans le contexte de la crise qui frappe le monde capitaliste et que reflète le roman contemporain (cf. l'incipit de Corazón tan blanco de Marías ou celui de La lluvia amarilla de Llamazares). Gilles Ernst ${ }^{9}$ rappelle que la crise actuelle de la mort qui frappe le monde occidental existe parce que la mort est devenue, comme naguère le sexe, ce qui ne se montre pas. Une désacralisation du mourir s'opère à partir de 1886, date de publication du roman de Tolstoï, $L a$ mort d'Ivan Ilitch, dans lequel se fait jour la solitude du mourant perdu dans l'indifférence de la foule. Cette désacralisation s'est accentuée pendant la première moitié du $\mathrm{XX}^{\mathrm{e}}$ siècle, pour devenir une crise sans précédent au cours des trente dernières années ${ }^{10}$ : elle va de pair avec un culte du corps.

${ }^{9}$ Gilles ERNST, Georges Bataille. Analyse du récit de mort, Paris, PUF, 1993, p. 16.

${ }^{10}$ Marie-Jeanne LECLERC, La mort et le récit, Montpellier, Centre de Recherches en Littérature Générale et Comparée de l'Université Paul Valéry, 1990. 